\title{
Exploring the magnetic topologies of cool stars
}

J. Morin ${ }^{1,2}$, J.-F. Donati ${ }^{2}$, P. Petit ${ }^{2}$, L. Albert ${ }^{3}$, M. Auriére ${ }^{2}$, R. Cabanac ${ }^{2}$, C. Catala ${ }^{4}$, X. Delfosse ${ }^{5}$, B. Dintrans ${ }^{2}$, R. Fares ${ }^{2}$, T. Forveille ${ }^{5}$, T. Gastine ${ }^{2}$, M. Jardine ${ }^{7}$, R. Konstantinova-Antova ${ }^{6}$, J. Lanoux ${ }^{8}$, F. Lignires ${ }^{2}$, A. Morgenthaler ${ }^{2}$, F. Paletou ${ }^{2}$, J.C. Ramirez Velez ${ }^{4}$, S.K. Solanki ${ }^{9}$, S. Thado ${ }^{2}$ and V. Van Grootel ${ }^{2}$

${ }^{1}$ Dublin Institute for Advanced Studies, 31 Fitzwilliam Place, Dublin 2, Ireland email: jmorin@cp.dias.ie

${ }^{2}$ LATT, Universit de Toulouse, CNRS, 14 Av. E. Belin, 31400 Toulouse, France

${ }^{3}$ CFHT, 65-1238 Mamalahoa Hwy, Kamuela HI 96743, USA

${ }^{4}$ LESIA, Observatoire de Paris-Meudon, 92195 Meudon, France

${ }^{5}$ LAOG, UMR5571 CNRS, Universit Joseph Fourier, BP 53, 38041 Grenoble, France

${ }^{6}$ Institute of Astronomy, Bulgarian Academy of Sciences, 72 Tsarigradsko shose, Sofia, Bulgaria

${ }^{7}$ School of Physics and Astronomy, University of St Andrews, St Andrews, Scotland KY16 9SS

${ }^{8}$ Centre dEtude Spatiale des Rayonnements, Universit de Toulouse, CNRS, France

${ }^{9}$ Max-Planck Institut fr Sonnensystemforschung, Katlenburg-Lindau, Germany

\begin{abstract}
Magnetic fields of cool stars can be directly investigated through the study of the Zeeman effect on photospheric spectral lines using several approaches. With spectroscopic measurement in unpolarised light, the total magnetic flux averaged over the stellar disc can be derived but very little information on the field geometry is available. Spectropolarimetry provides a complementary information on the large-scale magnetic topology. With Zeeman-Doppler Imaging (ZDI), this information can be retrieved to produce a map of the vector magnetic field at the surface of the star, and in particular to assess the relative importance of the poloidal and toroidal components as well as the degree of axisymmetry of the field distribution.

The development of high-performance spectropolarimeters associated with multi-lines techniques and ZDI allows us to explore magnetic topologies throughout the Hertzsprung-Russel diagram, on stars spanning a wide range of mass, age and rotation period. These observations bring novel constraints on magnetic field generation by dynamo effect in cool stars. In particular, the study of solar twins brings new insight on the impact of rotation on the solar dynamo, whereas the detection of strong and stable dipolar magnetic fields on fully convective stars questions the precise role of the tachocline in this process.
\end{abstract}

Keywords. Stars: low-mass, brown dwarfs, stars: magnetic fields, stars: activity, stars: rotation, techniques: spectroscopic, techniques: polarimetric

\section{Context: stellar dynamos}

Magnetic field is a key parameter to understand stellar formation and evolution. In cool stars, it powers activity phenomena that are observed across a large part of the electromagnetic spectrum and a wide range of timescales. Since the early $\mathrm{XX}^{\text {th }}$ century, the cyclic solar magnetic field has been thought to be constantly regenerated against ohmic dissipation by a magnetohydrodynamical process: the dynamo. Although the solar dynamo is still far from being fully understood, the basic concepts, as exposed by Parker (1955) in his $\alpha \Omega$ dynamo model are rather simple: (i) an initially poloidal magnetic 
field is converted into a stronger toroidal one by differential rotation, (ii) a poloidal field component is regenerated from the toroidal field by a second mechanism, such as the $\alpha$ effect. Two decades ago, helioseismology revealed the existence of the tachocline (e.g., Spiegel \& Zahn 1992), a thin layer of strong shear located at the interface between the inner radiative zone and the convective envelope. Since then, many theoretical and numerical studies have stressed the crucial role of the tachocline in the solar dynamo, being the place where large-scale toroidal fields can be stored and strongly amplified (e.g., Charbonneau \& MacGregor 1997).

Partly convective cool stars possess an internal structure similar to that of the Sun, i.e. an inner radiative zone and an outer convective envelope supposedly separated by a tachocline. Hence, it is generally assumed that their magnetic fields - as revealed by activity or direct measurements - are generated by a solar-like dynamo. However, some cool partly-convective stars strongly differ from the Sun, either in depth of their convective zone or rotation rate, and the impact of these differences on their dynamo is mostly unknown. On the other hand, main sequence stars less massive than $\sim 0.35 \mathrm{M}_{\odot}$ $(\sim \mathrm{M} 3)$ are fully convective (e.g., Chabrier \& Baraffe 1997) and therefore do not possess a tachocline. If the tachocline is indeed an essential part of the solar dynamo, magnetic field generation in these fully convective objects must rely on different physical processes.

\section{Magnetic field measurement and modelling}

Direct measurements of stellar magnetic fields rely on the properties of the Zeeman effect. Two complementary methods are successfully applied to cool stars. By measuring Zeeman broadening of photospheric spectral lines it is possible to assess the magnetic flux averaged over the visible stellar disc (e.g., Saar 1988). This method is therefore able to probe magnetic fields regardless of their complexity but provides virtually no information about the field geometry. On its part, the analysis of Zeeman polarisation in spectral lines provides information on the vector properties (i.e. strength, orientation and polarity). However, as neighboring magnetic regions of opposite polarities result in polarised signatures of opposite sign that cancel each other when integrating over the stellar disc, spectropolarimetry can only detect the large-scale component of stellar magnetic fields.

Polarised signatures in spectral lines of cools active stars have a small amplitude, making their measurement a hard task. Two advances have brought a large number of cool stars within reach of spectropolarimetric measurements. First, multi-line techniques (such as LSD, Donati et al. 1997) extract the polarimetric information from a large number of photospheric lines resulting in a $\mathrm{S} / \mathrm{N}$ multiplex gain that can reach as high as several tens when thousands of lines are used. Secondly, the new generation spectropolarimeters ESPaDOnS and NARVAL (see Donati 2003) feature a high overall efficiency and cover the full optical domain allowing to take full advantage of multi-line techniques.

Thanks to $(i)$ the sensitivity of the Zeeman effect to field lines orientation $(i i)$ rotational modulation and (iii) Doppler effect, the temporal evolution of polarised signatures in stellar lines strongly characterizes the parent magnetic topology. Thus, from a timeseries of circularly polarised spectra sampling the stellar rotation cycle, Zeeman-Doppler Imaging can perform a maximum entropy reconstruction of the vector magnetic field distribution at the surface of the star (Semel 1989; Donati \& Brown 1997). Although the resulting magnetic map has a better resolution for high $v \sin i$ values, this technique is also successfully applied to slow rotators (e.g., Morin et al. 2008b). For high S/N data 




Figure 1. Rotational dependence of the mean reconstructed magnetic flux (green line), and of the fraction of magnetic energy stored in the poloidal field component (red line).

spanning several stellar rotations, differential rotation can also be constrained (e.g., Petit et al. 2002).

\section{Solar twins}

We selected a sample of 4 nearby dwarfs with stellar parameters as close as possible to the solar ones, except for the rotation period (see Petit et al. 2008). In particular, their internal structure is expected to be very similar to the Sun's. We aim at studying the effect of rotation alone on the solar dynamo. For each star, a set of $\sim 10$ pairs of unpolarised and circularly polarised spectra was collected with NARVAL, from which we can map the surface magnetic field and to precisely determine the rotation period.

The first conclusion of this study is that the shorter the rotation period, the stronger is the large-scale magnetic field (see Fig. 1, green line). For the 2 slow rotators $\left(P_{\text {rot }}=\right.$ 22.7 and $20.5 \mathrm{~d}$ ), the magnetic maps reconstructed by ZDI are dominated by low order multipole modes, this is reminiscent of the solar global magnetic field. The fast rotators $\left(P_{\text {rot }}=12.3\right.$ and $8.8 \mathrm{~d}$ ) mainly feature a strong belt of toroidal field roughly encircling the pole, similar to the magnetic topologies of very active cool stars (e.g., Donati et al. 2003). The transition from an almost purely poloidal magnetic field to a strongly toroidal one, is apparently due to rotation, with a $P_{\text {rot }}$ threshold located between 12 and $20 \mathrm{~d}$ (see Fig. 1). These observations are in agreement with recent MHD simulations of solartype stars where dynamo action produces mostly toroidal magnetic topologies, featuring strong belts throughout the bulk of the convective envelope for $\Omega=3 \Omega_{\odot}$ (Brown et al. (2010), although the simulation domain does not encompass the stellar surface).

From our unpolarised spectra we measure the Ca II activity, and find that the $R_{H K}^{\prime}\left(B_{\text {mean }}\right)$ relation follows a power-law with an exponent of 0.32 . This is significantly different from the solar relation (established from observations of the quiet Sun and active regions) which has an exponent of 0.6 (e.g., Schrijver et al. 1989). As the chromospheric flux is also sensitive to spatial scales smaller than those contributing to the polarimetric 
signal, this apparent discrepancy suggests that a larger fraction of the total magnetic energy lies in the large-scale component as rotation increases.

The stars we have observed are expected to exhibit magnetic cycles and associated evolution of their topology. Chromospheric activity monitoring exists for two stars of our sample (e.g., Hall et al. 2007), showing in particular that our slowest rotator ( $P_{\text {rot }}=$ $22.7 \mathrm{~d}$ ) undergoes an activity cycle of $7 \mathrm{yr}$, and that we observed it in a high activity state. The predominantly quadrupolar magnetic topology we reconstruct is indeed reminiscent of the Sun's topology close to solar maximum (Sanderson et al. 2003). On the fastest rotator $\left(P_{\text {rot }}=8.8 \mathrm{~d}\right.$ ) we observe strong year-to-year evolution: between 2007 and 2008 the polarity of the main ring of azimuthal field had its polarity reversed, and between 2008 and 2009 the fraction of magnetic energy stored in poloidal field dramatically increased (see Petit et al. 2009). These rapid changes suggest a short cycle, and therefore a correlation: faster rotation would imply shorter activity cycles. Spectropolarimetric observations of the rapid rotator $\tau$ Boo $\left(P_{\text {rot }}=3.3 \mathrm{~d}\right)$ by Fares et al. $(2009)$ have also revealed two polarity reversals of the poloidal field component in two years, although in this case the higher stellar mass $\left(M_{\star}=1.3 \mathrm{M}_{\odot}\right)$ and the close-in orbiting giant planet may also play a role.

\section{Fully convective stars}

Following the first detection in polarised light of a large-scale magnetic field on a fully convective star by Donati et al. (2006), we have carried out the first spectropolarimetric survey of a sample $\mathrm{M}$ dwarfs lying on both sides of the fully convective boundary $(0.08<$ $\left.M_{\star}<0.75 \mathrm{M}_{\odot}\right)$ and spanning a wide range of periods $\left(0.33<P_{\text {rot }}<18.6 \mathrm{~d}\right)$. A total of 23 stars were selected, all are active so that we can supposedly detect polarised signatures and map their surface magnetic field. For each star we collected one or more time-series of unpolarised and circularly polarised spectra. We could reconstruct a map of the largescale surface magnetic field and derive an accurate period measurement of 18 stars. For the 5 remaining stars some constraints about the magnetic topology and an upper limit for the rotation period could still be derived. For more details see Donati et al. (2008) and Morin et al. (2008a,b, 2010).

The main results of this study are presented on Fig. 2, presenting the main properties of the reconstructed magnetic topologies as a function of stellar mass and rotation period. Our analysis reveals 3 distinct groups in this diagram.

(a) $\mathrm{M}$ dwarfs more massive than $\sim 0.5 \mathrm{M}_{\odot}$ (partly convective) exhibit magnetic topologies with a strong toroidal component, even dominant in some cases; the poloidal component is strongly non-axisymmetric. For most of these stars, we can measure surface differential rotation, values are between 60 and $120 \mathrm{mrad} \mathrm{d}^{-1}$ (i.e. between once and twice the solar rate approximately), and the topologies evolve beyond recognition on a timescale of a few months. These properties are reminiscent of the observations of more massive ( $\mathrm{G}$ and $\mathrm{K}$ ) active stars (e.g., Donati et al. 2003).

(b) Stars with masses between $\sim 0.2$ and $0.5 \mathrm{M}_{\odot}$ (close the fully convective limit) host much stronger large-scale magnetic field with radically different topologies: almost purely poloidal, generally nearly axisymmetric, always close to a dipole more or less tilted with respect to the rotation axis. These topologies are observed to be stable on timescales of several years, and differential rotation (when measurable) is of the order or a tenth of the solar rate. Our findings are in partial agreement with the recent numerical study by Browning (2008). Similarly, we observe that fully convective stars can generate strong and long-lived large-scale magnetic fields featuring a strong axisymmetric component, that are able to quench differential rotation. But we observe almost purely poloidal surface 


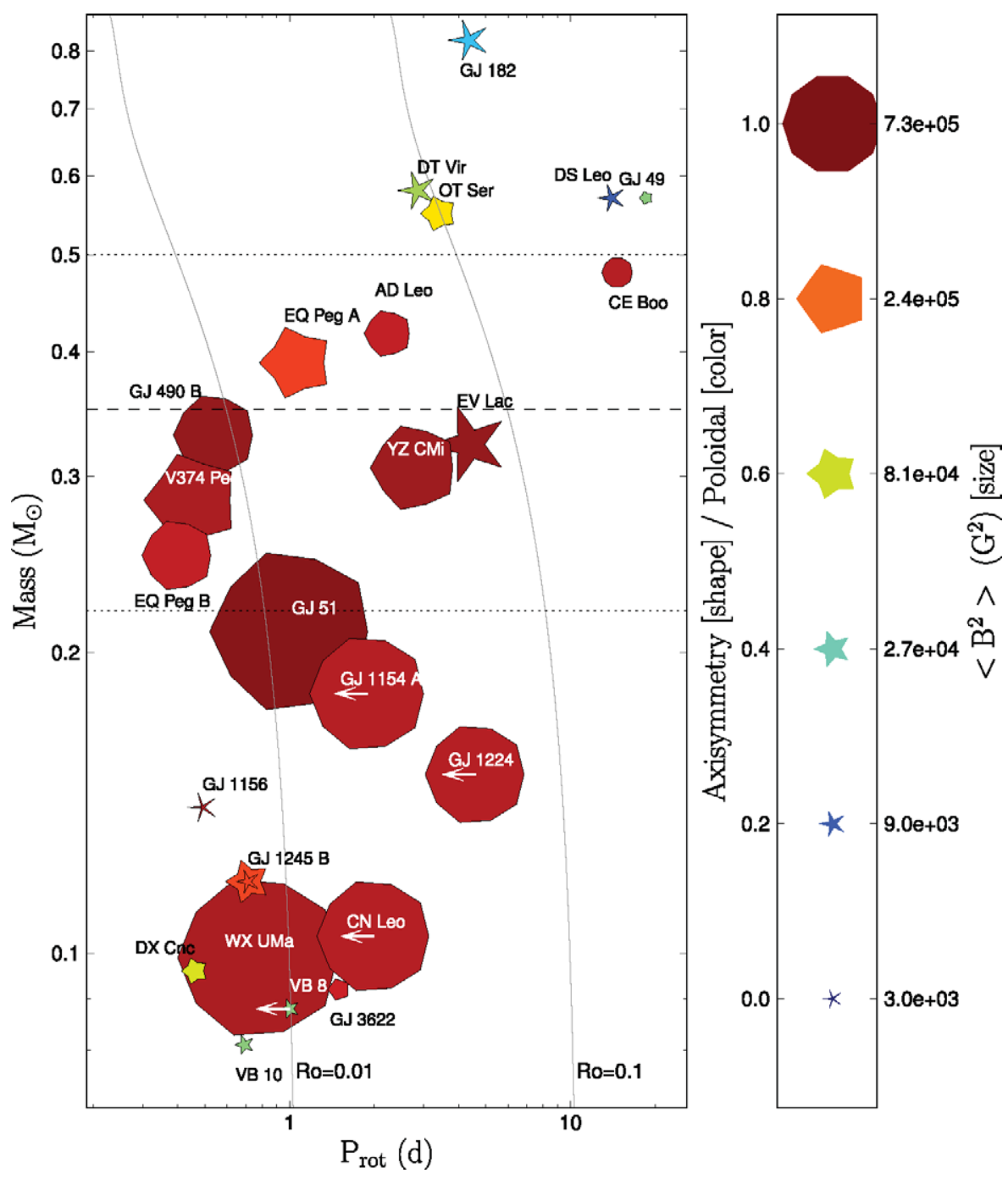

Figure 2. Properties of the magnetic topologies of our sample of M dwarfs (plus GJ 490 B, Phan-Bao et al. 2009) as a function of rotation period and mass. Larger symbols indicate stronger fields, symbol shapes depict the degree of axisymmetry of the reconstructed magnetic field (from decagons for purely axisymmetric to sharp stars for purely non axisymmetric), and colours the field configuration (from blue for purely toroidal to red for purely poloidal). Solid lines represent contours of constant Rossby number $R o=0.1$ (saturation threshold) and 0.01 . The theoretical full-convection limit $\left(M_{\star} \sim 0.35 \mathrm{M}_{\odot}\right)$ is plotted as a horizontal dashed line, and the approximate limits of the three stellar groups discussed in the text are represented as horizontal dotted lines.

magnetic fields, whereas in the simulation the axisymmetric component of the field is mainly toroidal (although the simulation does not encompass the stellar surface).

(c) Below $\sim 0.2 \mathrm{M}_{\odot}$, we observe 2 different categories of magnetic fields: either a very strong dipole (similar to group $b$ above) or a much weaker field generally featuring a significant non-axisymmetric component, and in some cases a toroidal one. Strong temporal variability is also observed on some objects of this second category. However stars in both categories have similar stellar parameters and cannot be separated in the mass-rotation diagram. This unexpected observation is not yet understood, and may be explained in 
several different ways: for instance, another parameter than mass and rotation period (such as age) may be relevant, two dynamo modes may be possible or stars may switch between two states in this mass range, etc.

\section{Conclusions and future work}

Taking advantage of recent developments in spectropolarimetric instrumentation and analysis techniques, it is nowadays possible to study dynamo action across the whole cool stars regime. We focus here on two projects: solar twins and fully convective M dwarfs. Both studies have already provided dynamo theorists with novel observational constraints, and have also raised new questions on the impact of magnetic fields and their topology on e.g., stellar spindown, structure, and chromospheric and coronal activity.

Our understanding of stellar dynamos also benefits from spectropolarimetric studies targeting other objects than cool main sequence stars. For instance, the first results obtained on a few $\mathrm{T}$ Tauri stars located on both sides of the fully convective boundary reveal similarities with low-mass main sequence stars (e.g., Donati et al. 2010), while the spectropolarimetric discovery of a magnetic field on Betelgeuse suggests that veryslowly-rotating supergiants can sustain local dynamo action (Auriēre et al. 2010).

Ongoing and future work includes in particular long-term monitoring of a small sample of selected targets to study magnetic cycles, and the extension of the survey to cover the mass-rotation plane on the whole cool stars regime.

\section{Acknowledgements}

The presentation of this paper in the IAU Symposium 273 was possible due to partial support from the National Science Foundation grant numbers ATM 0548260, AST 0968672 and NASA - Living With a Star grant number 09-LWSTRT09-0039.

\section{References}

Auriēre M., et al., 2010, Astron. Astrophys, 516, L2

Brown B. P., Browning M. K., Brun A. S., Miesch M. S., Toomre J., 2010, Astrophys. J., 711, 424

Browning M. K., 2008, Astrophys. J., 676, 1262

Chabrier G., Baraffe I., 1997, Astron. Astrophys, 327, 1039

Charbonneau P., MacGregor K. B., 1997, Astrophys. J., 486, 502

Donati J.-F., Semel M., Carter B. D., Rees D. E., Cameron A. C., 1997, Mon. Not. Roy. Astron. Soc., 291, 658

Donati J.-F. \& Brown S. F., 1997, Astron. Astrophys, 326, 1135

Donati J.-F., et al., 2003, Mon. Not. Roy. Astron. Soc., 345, 1145

Donati J.-F., 2003, ASPC, 307, 41

Donati J.-F., et al., 2006, Science, 311, 633

Donati J.-F., et al., 2008, Mon. Not. Roy. Astron. Soc., 390, 545

Donati J.-F., et al., 2010, Mon. Not. Roy. Astron. Soc., 402, 1426

Fares R., et al., 2009, Mon. Not. Roy. Astron. Soc., 398, 1383

Hall J. C., Lockwood G. W., \& Skiff B. A., 2007, AJ, 133, 862

Morin J., et al., 2008a, Mon. Not. Roy. Astron. Soc., 384, 77

Morin J., et al., 2008b, Mon. Not. Roy. Astron. Soc., 390, 567

Morin J., Donati J.-F., Petit P., Delfosse X., Forveille T., \& Jardine M. M., 2010, Mon. Not. Roy. Astron. Soc., 407, 2269

Parker E. N., 1955, Astrophys. J., 122, 293

Petit P., Donati J.-F., Collier Cameron A., 2002, Mon. Not. Roy. Astron. Soc., 334, 374

Petit P., et al., 2008, Mon. Not. Roy. Astron. Soc., 388, 80 
Petit P., et al., 2009, Astron. Astrophys, 508, L9

Phan-Bao N., Lim J., Donati J.-F., Johns-Krull C. M., \& Martín E. L., 2009, Astrophys. J., 704,1721

Saar S. H., 1988, Astrophys. J., 324, 441

Sanderson T. R., Appourchaux T., Hoeksema J. T., \& Harvey K. L., 2003, JGRA, 108, 1035

Schrijver C. J., Cote J., Zwaan C., \& Saar S. H., 1989, Astrophys. J., 337, 964

Semel M., 1989, Astron. Astrophys, 225, 456

Spiegel E. A. \& Zahn J.-P., 1992, Astron. Astrophys, 265, 106 